\title{
The Impact of Telemedicine on Quality of Care for Patients with Diabetes After March 2020
}

\author{
Jacob K. Quinton, MD, MSHS ${ }^{\top}$, Michael K Ong, MD, $P h D^{1,4,5}$, \\ Catherine Sarkisian, $M D, M S H S^{1,3}$, Alejandra Casillas, $M D, M S H S^{7}$, Sitaram Vangala, $\mathrm{MS}^{7}$, \\ Preeti Kakani, $B S^{2}$, and Maria Han, $M D, M S H S^{7}$
}

\begin{abstract}
'Division of General Internal Medicine and Health Services Research, Department of Medicine, University of California, 1100 Glendon Ave, Suite 900, Los Angeles, CA, USA; ${ }^{2}$ David Geffen School of Medicine, University of California, Los Angeles, Los Angeles, CA, USA; ${ }^{3}$ Geriatrics Research Education and Clinical Center, Veterans Affairs Greater Los Angeles Healthcare System, Los Angeles, CA, USA; ${ }^{4}$ Department of Health Policy \& Management, Fielding School of Public Health, University of California, Los Angeles, Los Angeles, CA, USA; ${ }^{5}$ VA Center for the Study of Healthcare Innovation, Implementation and Policy (CSHIIP), Los Angeles, CA, USA.
\end{abstract}

BACKGROUND: The impact of telemedicine on ambulatory care quality is a key question for policymakers as they navigate payment reform for remote care.

OBJECTIVE: To evaluate whether utilizing telemedicine in the first 9 months of the COVID-19 pandemic impacted performance on a diabetes quality of care measure for patients at a large academic medical center. We hypothesized care quality would reduce less among telemedicine users.

DESIGN: Quasi-experimental design using binomial logistic regression. Covariates included age, gender, race, ethnicity, type of insurance, hierarchical condition category score, primary language at the individual level, and zip code-level income.

PARTICIPANTS: All adult patients younger than 75 years of age diagnosed with type 2 diabetes mellitus $(N=16,588)$ as of 3/19/2020 at a single academic health center.

INTERVENTIONS: Completion of one or more telemedicine encounters with an institutional primary care physician or endocrinologist between 3/19/2020 and 12/19/2020.

MAIN MEASURES: The components met in a five-item composite measure of diabetes quality of care, as of patients' last clinical encounter. Items were (1) systolic blood pressure less than $140 \mathrm{mmHg}$, (2) hemoglobin Alc less than $8.0 \%$, (3) using a statin and (4) aspirin, and (5) tobacco non-use.

KEY RESULTS: From the pre- to post-period, the probability of meeting any given component of the composite measure for patients only utilizing in-person care was $21 \%$ lower (OR, 95\% CI 0.79; 0.76, 0.81) and for the telemedicine users $2 \%$ lower (OR 0.98; 0.85, 1.13). There was an increased likelihood of meeting any given

Key Points

1. Question: How has the use of telemedicine since March 2020 impacted quality of care for chronic diseases like diabetes?

2. Findings: Since implementation of telemedicine in March 2020, patients with diabetes utilizing telemedicine in addition to in-person care achieved similar quality outcomes, while the quality outcomes for patients utilizing only in-person care declined.

3. Meaning: Use of telemedicine in addition to in-person care maintained quality of care for patients with diabetes in the early pandemic at one academic center

Received August 4, 2021

Accepted December 16, 2021

Published online January 28, 2022 component among telemedicine users compared to inperson care alone (OR 1.25; 1.08, 1.44).

CONCLUSIONS: Patients with diabetes utilizing telemedicine performed similarly on a composite measure of diabetes care quality compared to before the pandemic. Those not utilizing telemedicine had reductions. Telemedicine use maintained quality of care for patients with diabetes during the first 9 months of the COVID-19 pandemic.

J Gen Intern Med 37(5):1198-203

DOI: $10.1007 / \mathrm{s} 11606-021-07367-3$

This is a U.S. government work and not under copyright protection in the U.S.; foreign copyright protection may apply 2022. This article is an open access publication

\section{BACKGROUND}

The coronavirus disease 19 (COVID-19) pandemic and the subsequent public health emergency (PHE) triggered a more than twenty-fold increase in telemedicine utilization over a 2week period in March 2020 in order to maintain access to services while mitigating the threat of possible COVID-19 transmission. As in-person visits sharply declined, the proportion of ambulatory care delivered via telemedicine (defined as both audio-only and audio-video encounters) increased to peak at nearly half of all ambulatory encounters in June $2020 .^{1}$ Subsequently, telemedicine encounters fell with the resumption of in-person care, but telemedicine visit volume persisted at nearly a quarter of total ambulatory encounters for the first 9 months of the pandemic. This "blend" of in-person and remote care in the ambulatory setting transformed office-based medical practice. The durability of this blended model of ambulatory care will depend on not just the changes in access, ${ }^{1-4}$ possible exacerbations of disparities, ${ }^{5-9}$ but also whether or not remote care is of the same quality as care delivered in person.

The expansion of telemedicine during the PHE was supported by many temporary waivers of telemedicine regulations by the Centers for Medicare and Medicaid Services (CMS) to increase capacity, expand workforce, reduce administrative 
burden, and otherwise expand telemedicine services. ${ }^{10}$ Telehealth has been previously demonstrated to improve chronic disease management ${ }^{11-14}$ for small pilots of patients with diabetes. A widely cited meta-analysis included more than forty randomized trials ${ }^{15-18}$ mostly used telemonitoring. ${ }^{19}$ Broad populations have been studied including a randomized intervention considering quality of care domains for veterans, ${ }^{20}$ and among older, racially diverse populations. ${ }^{13}$ Despite this evidence until the COVID-19-related PHE, there has not been broad, population-wide telemedicine utilization, and previous studies were subject to the generalizability issues inherent in smaller demonstrations.

The Medicare Payment Advisory Commission (MedPAC) has recommended continuation of coverage for telemedicine for 1-2 years after the end of the PHE in order to gather data on access, quality, and cost of care, ${ }^{21}$ which was recently incorporated in the most recent CMS physician fee scale. ${ }^{22}$ Our study objective was to evaluate the impact of remote audio-video encounter utilization on the quality of care for patients with diabetes at a large academic medical center in the first 9 months of the PHE. ${ }^{23}$

\section{METHODS}

\section{Data Sources}

We analyzed electronic medical record (EMR) data for all patients who were identified by our institutional population registry as having type 2 diabetes mellitus (DM) as of March 19, 2020. We abstracted the following variables from our institution's electronic medical record (EMR) at the individual level: decade of age, gender, race, ethnicity, category of insurance (Medicare, commercial, Medicaid, or institutional managed care plan), systolic blood pressure, hemoglobin A1c, aspirin and/or statin prescription, smoking status, ambulatory visits to primary care, endocrinology, and other department of medicine visits, as well as hierarchical condition category (HCC) score. We also included income information from the American Communities Survey 2018 5-year estimates at the five-digit zip code level.

\section{Design and Study Period}

We used a quasi-experimental (difference-in-differences) design. We defined our post-period to include the 9 months following the declaration of "safer-at-home" orders in Los Angeles County on March 19, 2020. ${ }^{24}$ We defined two sequential pre-periods of the same duration in the 18 months prior to the declaration to identify if pre-pandemic quality of care trends were parallel.

\section{Study Population and Exclusion Criteria}

Our study population $(N=21,460)$ consisted of all patients identified by institutional clinicians as having type 2 diabetes mellitus when added to the electronic medical record problem list. This is verified for accuracy by the institutional quality team. Two physicians, one registered nurse, and two nonclinicians independently verify a random sample of patients identified as having type 2 diabetes mellitus. The study population was restricted to align with quality reporting standards, restricting to include adult patients under age 75 who are not currently enrolled in hospice care $(N=16,588)$.

\section{Study Variables}

Quality Outcome. We defined our outcome for quality of care for patients with diabetes using the same domains as indicators included in the CMS Diabetes Composite quality measure from the CMS Measures Inventory. ${ }^{25}$ For our primary outcome, we evaluated the probability of meeting any given component in the five-component composite measure. The components are the last recorded value of (1)systolic/diastolic blood pressure less than 140/90 mmHg, (2) hemoglobin A1c less than $8.0 \%$, (3) active prescription for statins and/or(4) aspirin or other antiplatelet agents if not contraindicated, and (5) tobacco non-users or those that quit during the study period. ${ }^{23}$ We made prespecified changes to the composite for the purposes of our analysis. Due to limitations in our EMR, we did not exclude patients who may have a contraindication for anti-platelets or consider other anti-platelet agents (i.e., clopidogrel) as meeting this measure criterion, and did not analyze diastolic blood pressure as the proportion of patients with diastolic-only hypertension is negligible. We also did not exclude patients who became pregnant during the study period and were not able to ascertain deaths in our cohort.

Exposure and Control Group. We identified all included patients with at least one telemedicine visit in the post-period to either a primary care clinician $(N=6,405)$ or an endocrinologist $(N=2,251)$ to define our total exposure group $(N=$ $7,581)$. Telemedicine visits are defined at our institution using the (EPIC/Clarity) visit types as recorded in our EMR, and distinguish between telephone encounters, new video visits, and return video visits. Visit type codes are unique to the EMR instance but available on request.

Covariates. We included the following covariates in addition to our primary regressor: decade of age, gender, race, ethnicity, category of insurance (Medicare, commercial, Medicaid, or institutional managed care plan), hierarchical condition category (HCC) score, all at the individual level. We also included income information from the American Communities Survey 2018 5-year estimates at the five-digit zip code level.

Our study protocol was approved by the UCLA Institutional Review Board via expedited review.

\section{Statistical Analysis}

Using a binomial regression model, we estimated the effect of telemedicine use (at least one encounter in primary care or endocrinology) by comparing the likelihood of meeting any 
individual component of the diabetes composite outcome in the 9 months before and after March 2020 between the exposure and control group. All models included as covariates the sociodemographic and clinical characteristics described above. We performed several sensitivity analyses including (1) meeting all five component indicators of the diabetes composite measure, and sequentially fewer $(4 / 5,3 / 5,2 / 5$, and 1/5) indicators, (2) meeting four component indicators omitting the systolic blood pressure variable, which we hypothesized would be difficult to assess via telemedicine, and (3) a subgroup analysis of patients receiving Medicare benefits (either through disability or over the age of 65 years) and meeting $4 / 5$ indicators given hypothesized challenges of older and disabled patients in accommodating new technology (Appendix 1). As a fourth sensitivity analysis to determine if there was a disproportionate quality impact on complex patients, we performed a subgroup analysis of patients meeting $4 / 5$ outcome indicators among those with an HCC score of two or above, double that of the typical Medicare beneficiary, approximately in the top decile of complexity for our population (Appendix 1).

A significance level of 0.05 was used throughout, and analyses were performed using Stata 16c (StataCorp, College Station, TX). Our binomial regression model was specified by using the STATA command "binreg", and all sensitivity analyses were using logistic regression ("logistic").

\section{RESULTS}

Very few patients with diabetes utilized telemedicine before the pandemic $(N=224,1 \%)$ and most patients utilizing telemedicine in the post-period also had in-person appointments $(6292,86 \%)$. Both audio-only utilization $(2,784,17 \%)$ and audio-video utilization $(7,357,44 \%)$ increased dramatically in the post-period. Of audio-only utilizers, $66 \%$ were exclusively audio-only (i.e., no video encounters) telemedicine utilizers in the post-period. Telemedicine utilizers varied from those not utilizing telemedicine as less likely to have commercial insurance, more likely to be insured through Medicare and have lower HCC scores (Table 1). Nearly half of patients with diabetes utilized telemedicine during the first 9 months of the pandemic $(N=7,357,44 \%)$. These patients were more likely to be female, Latino/a, more likely to be insured through Medicare and an institutional managed care plan, and have lower HCC scores (Table 1 and Fig. 1).

Both groups had similar overall ambulatory utilization before and during the first 9 months of the pandemic (Appendix 2 Table 1). The proportion of patients meeting each component indicator of the overall diabetes composite measure were similar in the 18 months prior to the pandemic (Appendix 2, Fig. 1).

In adjusted estimates, patients utilizing in-person care alone in the first 9 months of the pandemic were less likely to meet any given component of the diabetes composite quality measure compared to before the pandemic (OR, 95\% CI; $0.60,0.56,0.65)$, whereas patients utilizing telemedicine were no less likely (OR, 95\% CI; 0.89, 0.65, 1.23). The interaction between time period and use of telemedicine services was significant (OR, 95\% CI; 1.48, 1.07, 2.05), as well as the individual-level covariates age (by decade of life), gender, self-reported Asian or other race, and the zip code-level covariate income (by federal poverty level) (Table 2).

Patients utilizing telemedicine were as likely than those not using telemedicine to meet 5 of 5 components of the diabetes composite measure of quality of care when comparing the post-period to pre-period (OR, 95\% CI; 1.27, 0.93, 1.73). Similarly, patients utilizing telemedicine were as likely as those using telemedicine to meet the remaining quality indicators when the blood pressure measurement component of the composite was excluded $(1.21,0.89,1.66)$ (Appendix 1). Among the subgroup analysis of Medicare beneficiaries' meeting 4 of 5 indicators $(N=9,264)$, we saw significant declines (OR $0.60 ; 0.54,0.67)$ in both the in-person care and telemedicine utilizing groups $(0.64 ; 0.41,1.01)$ with nonsignificant differences between the groups $(1.07 ; 0.68,1.70)$. Similarly, among patients with an HCC score of 2 or more $(N$ $=5,766)$, we observed a reduction in those meeting 4 of 5 indicators among patients receiving in-person care alone $(0.45 ; 0.37,0.55)$ although not among those patients utilizing telemedicine services $(0.86 ; 0.38,2.00)$, and the difference between the groups was not statistically significant (OR $1.91 ; 0.80,4.57)$. Few patients with an HCC score of 2 or more utilized telemedicine $(N=946)$. Full covariate estimates for sensitivity analyses are available in a supplementary appendix (Appendix 1).

\section{DISCUSSION}

Telemedicine has been shown to impact the quality of care for patients with diabetes in pre-pandemic demonstrations, but however has not previously been implemented across entire populations. Patients with diabetes in a large academic medical center who used telemedicine achieved similar quality outcomes compared to before the pandemic; however, patients who utilized only in-person care saw a decline in the quality outcome ascertainment. We found this to be the case using a composite indicator of quality of care for diabetes in the CMS measures inventory, despite care of similar quality during the 18 months before the pandemic, while controlling for an array of sociodemographic and clinical variables in our analysis. This demonstration of the incorporation of telemedicine as a mode of ambulatory care in addition to in-person visits during the early pandemic is an early indicator of the promise of telemedicine to deliver high-quality care across populations, during and beyond the public health emergency.

The largest differences between the telemedicine utilizing and the in-person care only group were seen in the indicators most likely to be impacted by a disruption of in-person care, 
Table 1 Characteristics of patients with diabetes utilizing telemedicine compared to in-person care alone, in the 9 months before and after the beginning of the COVID-19 pandemic

\begin{tabular}{|c|c|c|c|c|}
\hline \multirow[t]{2}{*}{ Characteristic } & \multicolumn{2}{|c|}{ Telemedicine utilizers } & \multicolumn{2}{|c|}{ In-person care alone } \\
\hline & $\begin{array}{l}\text { Pre-period } \\
\text { 6/19/19-3/19/20 }\end{array}$ & $\begin{array}{l}\text { Post-period } \\
\text { 3/19/20-12/19/20 }\end{array}$ & $\begin{array}{l}\text { Pre-period } \\
\text { 6/19/19-3/19/20 }\end{array}$ & $\begin{array}{l}\text { Post-period } \\
3 / 19 / 20-12 / 19 / 20\end{array}$ \\
\hline Individuals $(N)$ & 224 & 7,357 & 16,364 & 9,231 \\
\hline Age, mean (SD) & $60(11)$ & $60(11)$ & $60(11)$ & $60(11)$ \\
\hline Female $(\%)$ & $119(53)^{*}$ & $3,600(49) *$ & $7,348(45)^{*}$ & $3,867(42)^{*}$ \\
\hline \multicolumn{5}{|l|}{ Race $(\%)$} \\
\hline American Indian & $1(<1)$ & $38(1)$ & $92(1)$ & $55(1)$ \\
\hline Asian & $27(12)$ & $1,057(14)$ & $2,535(15)$ & $1,505(16)$ \\
\hline Black & $21(9)$ & $822(11)$ & $1,605(10)$ & $804(9)$ \\
\hline Other/unknown & $53(24)$ & $1,690(23)$ & $4,123(25)$ & $2,486(27)$ \\
\hline Pacific Islander & $0(0)$ & $25(<1)$ & $68(<1)$ & $43(<1)$ \\
\hline White & $122(54)$ & $3,722(51)$ & $7,935(49)$ & $4.335(47)$ \\
\hline Ethnicity (\% Latino/a) & $51(23)$ & $1,402(19) *$ & $2,996(18)$ & $1,634(18)^{*}$ \\
\hline \multicolumn{5}{|l|}{ Insurance type } \\
\hline Commercial & $86(39)^{*}$ & $3,136(43)^{*}$ & $7,584(48)^{*}$ & $4,534(52)^{*}$ \\
\hline Medicaid & $1(1)^{*}$ & $69(1)^{*}$ & $180(1)^{*}$ & $112(1)^{*}$ \\
\hline Medicare & $84(38) *$ & $2,530(35)^{*}$ & $5,059(32) *$ & $2,613(30)^{*}$ \\
\hline Other & $1(1)^{*}$ & $19(<1)^{*}$ & $38(1)^{*}$ & $20(1)^{*}$ \\
\hline Managed care & $47(21) *$ & $1,514(21)^{*}$ & $2,861(18)^{*}$ & $1,394(16)^{*}$ \\
\hline Primary language other than English (\%) & $10(5)$ & $497(7)$ & $1,185(7)$ & $698(8)$ \\
\hline \multicolumn{5}{|l|}{ HCC score } \\
\hline $0-1$ & $163(76)^{*}$ & $5,407(74) *$ & $11,379(70)^{*}$ & $6,135(66)^{*}$ \\
\hline $1-2$ & $29(14) *$ & $1,036(14) *$ & $1,681(10)^{*}$ & $674(7)^{*}$ \\
\hline $2-3$ & $10(5) *$ & $324(4)^{*}$ & $510(3)^{*}$ & $196(2)^{*}$ \\
\hline $3+$ & $12(6) *$ & $267(4) *$ & $386(2)^{*}$ & $131(1)^{*}$ \\
\hline Missing & $10(4) *$ & $323(4) *$ & $2,408(15)^{*}$ & $2,095(23)^{*}$ \\
\hline \multicolumn{5}{|l|}{ DM quality components } \\
\hline A1c less than $8 \%$ & $149(67)^{*}$ & $4,401(60)^{*}$ & $9,363(58)^{*}$ & $3,549(39)^{*}$ \\
\hline Systolic BP $<140$ & $166(76)^{*}$ & $4,228(58)^{*}$ & $10,227(63)^{*}$ & $3,639(40)^{*}$ \\
\hline Statin prescription & $178(95)$ & $5,788(96) *$ & $12,143(95)$ & $6,585(95)^{*}$ \\
\hline Aspirin prescription & $75(84)$ & $2,695(82)$ & $5,816(87)$ & $2,888(83)$ \\
\hline Tobacco non-use & $208(98)$ & $6,695(91)$ & $14,908(91)$ & $8,401(91)$ \\
\hline
\end{tabular}

$*_{p}<0.05$, comparisons between in-person care versus telemedicine users for each time period

i.e., blood pressure monitoring and laboratory testing (hemoglobin A1c), which may be improved with advances in remote patient monitoring. This disruption has been also seen in national cohorts. ${ }^{28}$ The unanswered question is whether continued innovations in telemedicine as a mode of ambulatory care, including at the authors' institution, ${ }^{26}$ will be able to

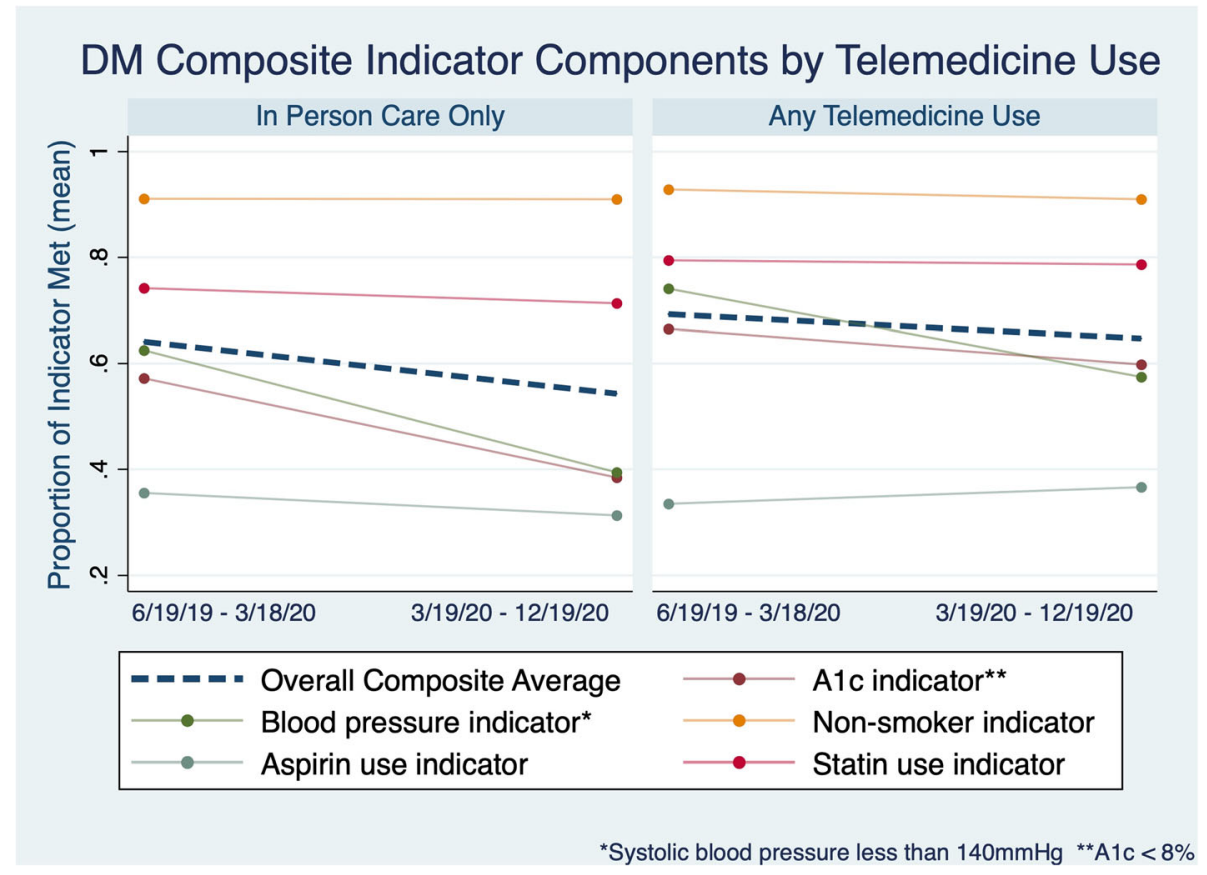

Figure 1 DM composite indicator components by Telemedicine use. 
Table 2 Odds of meeting diabetes quality composite components among patients with diabetes utilizing telemedicine versus in-person care alone $^{\wedge}$

\begin{tabular}{|c|c|c|c|}
\hline Characteristic & $\begin{array}{l}\text { Odds } \\
\text { ratio }\end{array}$ & $\begin{array}{l}\text { 95\% confidence } \\
\text { interval }\end{array}$ & pvalue \\
\hline $\begin{array}{l}\text { In-person care alone: post } \\
\text { versus pre-period }\end{array}$ & $0.60 *$ & $0.56-0.65$ & $<0.001$ \\
\hline $\begin{array}{l}\text { Any telemedicine use: post } \\
\text { versus pre-period }\end{array}$ & 0.89 & $0.65-1.23$ & 0.484 \\
\hline Difference-in-differences & $1.48 *$ & $1.07-2.05$ & 0.02 \\
\hline Age (decade) & $1.71^{*}$ & $1.66-1.78$ & $\begin{array}{l}< \\
0.001\end{array}$ \\
\hline Female gender & $0.58 *$ & $0.55-0.62$ & $<0.001$ \\
\hline \multicolumn{4}{|l|}{ Race or ethnic group } \\
\hline Black & 1.12 & $1.02-1.25$ & 0.024 \\
\hline Asian & $1.20 *$ & $1.10-1.30$ & $<.001$ \\
\hline Pacific Islander & 1.23 & $0.79-1.94$ & 0.36 \\
\hline American Indian & 0.81 & $0.54-1.21$ & 0.31 \\
\hline Other & $0.90^{*}$ & $0.83-0.97$ & 0.006 \\
\hline \multicolumn{4}{|l|}{ Insurance type } \\
\hline Medicare & 1.23 & $0.92-1.76$ & 0.15 \\
\hline Commercial & $1.15^{*}$ & $1.07-1.24$ & $<.001$ \\
\hline Managed care & $1.94 *$ & $1.10-3.43$ & 0.021 \\
\hline Medicaid & $1.11^{*}$ & $1.02-1.20$ & 0.008 \\
\hline Primary language & 0.76 & $0.68-0.85$ & 0.68 \\
\hline HCC score & $1.18^{*}$ & $1.13-1.22$ & $\begin{array}{l}< \\
0.001\end{array}$ \\
\hline
\end{tabular}

$\wedge$ Outcome is odds of meeting any additional quality indicator, binomial logistic regression, in the 9 months before and after the beginning of the COVID-19 pandemic

improve the quality of care for populations over time. Recent single-payer evidence highlights the role of payment models in this innovation. ${ }^{27}$

Our study is not without limitations. First, our data were from a single institution in a large urban environment, and limitations to the use of telemedicine (such as broadband access or technology limitations) may be less prevalent in our study population than in a predominantly low-income or rural population. Second, our intervention included all patients with at least one telemedicine encounter in the 9 months following the pandemic, and while this was the first opportunity to study population-wide telemedicine implementation, the time was uniquely transitional as "safer at home" orders were enacted, vaccines were developing, and case rates in our home county and state varied widely, perhaps leading to unobserved variable biases not controlled for in our detailed model. Third, part of this first population-wide implementation of telemedicine is the acknowledgement that despite observed variable consistency in diabetes care among preand early-pandemic telemedicine utilizers, the population using telemedicine before the pandemic may have varied from the general population at our institution on unobservable characteristics.

In conclusion, the rapid expansion of telemedicine utilization has transformed ambulatory care delivery during the COVID-19 pandemic, and improved access to care to populations attempting to minimize exposure to a deadly respiratory pathogen. This unprecedented change in the mode of ambulatory care delivery has many unanswered questions currently pressing policymakers. We found that in a single institution, the utilization in the 9 months following the COVID-19 pandemic improved the quality of care for patients with diabetes. Future studies are needed to validate these results in state- or nation-wide populations, and with additional chronic diseases, in order to make evidence-based policy regarding the continuation of telemedicine coverage after the public health emergency concludes.

Supplementary Information The online version contains supplementary material available at https://doi.org/10.1007/s11606-02107367-3.

Availability of Data and Materials: The datasets generated and/ or analyzed during the current study are not publicly available due to containing patient identifying information from electronic health record data, but are available from the corresponding author on reasonable request.

Corresponding Author: Jacob K. Quinton, MD, MSHS; Division of General Internal Medicine and Health Services Research, Department of Medicine, University of California, 1100 Glendon Ave, Suite 900, Los Angeles, CA 90024, USA (e-mail: jquinton@mednet.ucla.edu).

Author Contribution All authors contributed to the conception or design or acquisition, analysis or interpretation of data, or drafted or substantially revised the manuscript, and have approved the submitted version, and have agreed to be personally accountable for the authors' own contributions and to ensure that questions related to the accuracy or integrity of any part of the work are investigated, resolved, and resolution documented in literature. All authors have read and approved the manuscript.

\section{Declarations:}

Ethics Approval and Consent to Participate: Not applicable.

Consent for Publication: Not applicable.

Conflict of Interest: Dr. Michael Ong is the author of the UpToDate chapter on telemedicine and receives royalties for this authorship, outside of the submitted work.

\section{REFERENCES}

1. Patel SY, Mehrotra A, Huskamp HA, Uscher-Pines L, Ganguli I, Barnett ML. Trends in Outpatient Care Delivery and Telemedicine During the COVID-19 Pandemic in the US. JAMA Intern Med. 2021;181(3):388-391. https://doi.org/10.1001/jamainternmed.2020.5928

2. Patel SY, Mehrotra A, Huskamp HA, Uscher-Pines L, Ganguli I, Barnett ML. Variation In Telemedicine Use And Outpatient Care During The COVID-19 Pandemic In The United States. Health Aff. 2021;40(2):349358. https://doi.org/10.1377/hlthaff.2020.01786

3. Kakani P, Sorensen A, Quinton JK, et al. Patient Characteristics Associated with Telemedicine Use at a Large Academic Health System Before and After COVID-19. J Gen Intern Med. 2021;36(4):1166-1168. https://doi.org/10.1007/s11606-020-06544-0

4. Whaley CM, Pera MF, Cantor J, et al. Changes in Health Services Use Among Commercially Insured US Populations During the COVID-19 Pandemic. JAMA Netw Open. 2020;3(11):e2024984-e2024984. https:// doi.org/10.1001/jamanetworkopen.2020.24984

5. Cantor JH, McBain RK, Pera MF, Bravata DM, Whaley CM. Who Is (and Is Not) Receiving Telemedicine Care During the COVID-19 Pandemic. Am $J$ Prev Med. March 2021. https://doi.org/10.1016/j.amepre.2021.01. 030

6. Quinton JK, Han M, Casillas A, et al. Characteristics of Medicare beneficiaries utilizing telemedicine after July 2020. J Am Geriatr Soc. 2021. https://doi.org/10.1111/jgs. 17202 
7. Rodriguez JA, Saadi A, Schwamm LH, Bates DW, Samal L. Disparities In Telehealth Use Among California Patients With Limited English Proficiency. Health Aff. 2021;40(3):487-495. https://doi.org/10.1377/hlthaff. 2020.00823

8. Roberts ET, Mehrotra A. Assessment of Disparities in Digital Access Among Medicare Beneficiaries and Implications for Telemedicine. JAMA Intern Med. 2020;180(10):1386-1389. https://doi.org/10.1001/ jamainternmed.2020.2666

9. Eberly LA, Kallan MJ, Julien HM, et al. Patient Characteristics Associated With Telemedicine Access for Primary and Specialty Ambulatory Care During the COVID-19 Pandemic. JAMA Netw Open. 2020;3(12):e2031640-e2031640. https://doi.org/10.1001/ jamanetworkopen.2020.31640

10. Farb J, Care H, Yocom CL, Care H. MEDICARE AND COVID-19 Program Flexibilities and Considerations for Their Continuation. 2021.

11. Hanlon P, Daines L, Campbell C, Mckinstry B, Weller D, Pinnock H. Telehealth interventions to support self-management of long-term conditions: A systematic metareview of diabetes, heart failure, asthma, chronic obstructive pulmonary disease, and cancer. $J$ Med Internet Res. 2017;19(5):1-28. https://doi.org/10.2196/jmir.6688

12. Lee PA, Greenfield G, Pappas Y. The impact of telehealth remote patient monitoring on glycemic control in type 2 diabetes: A systematic review and meta-analysis of systematic reviews of randomised controlled trials BMC Health Serv Res. 2018;18(1):1-10. https://doi.org/10.1186/ s12913-018-3274-8

13. Shea S, Weinstock RS, Starren J, et al. A Randomized Trial Comparing Telemedicine Case Management with Usual Care in Older, Ethnically Diverse, Medically Underserved Patients with Diabetes Mellitus. J Am Med Informatics Assoc. 2006;13(1):40-51. https://doi.org/10.1197/ jamia.M1917

14. Zhai YK, Zhu WJ, Cai YL, Sun DX, Zhao J. Clinical- and costeffectiveness of telemedicine in type 2 diabetes mellitus: A systematic review and meta-analysis. Med (United States). 2014;93(28):e312. https://doi.org/10.1097/MD.0000000000000312

15. Timpel P, Oswald S, Schwarz PEH, Harst L. Mapping the Evidence on the Effectiveness of Telemedicine Interventions in Diabetes, Dyslipidemia and Hypertension: An Umbrella Review of Systematic Reviews and MetaAnalyses. J Med Internet Res. 2020;22(3):e16791. https://doi.org/10. 2196/16791

16. Wootton R. Twenty years of telemedicine in chronic disease managementan evidence synthesis. J Telemed Telecare. 2012;18(4):211-220. https:// doi.org/10.1258/jtt.2012.120219

17. Tchero H, Kangambega P, Briatte C, Brunet-Houdard S, Retali GR, Rusch E. Clinical Effectiveness of Telemedicine in Diabetes Mellitus: A MetaAnalysis of 42 Randomized Controlled Trials. Telemed e-Health.
2019;25(7):569-583. https://doi.org/10.1089/tmj.2018.0128

18. Polisena J, Tran K, Cimon K, Hutton B, McGill S, Palmer K. Home telehealth for diabetes management: A systematic review and metaanalysis. Diabetes, Obes Metab. 2009;11(10):913-930. https://doi.org/ 10.1111/j.1463-1326.2009.01057.x

19. Tchero H, Kangambega P, Briatte C, Brunet-Houdard S, Retali GR, Rusch E. Clinical Effectiveness of Telemedicine in Diabetes Mellitus: A MetaAnalysis of 42 Randomized Controlled Trials. Telemed e-Health. 2019;25(7):569-583. https://doi.org/10.1089/tmj.2018.0128

20. Lu AD, Gunzburger E, Glorioso TJ, et al. Impact of Longitudinal Virtual Primary Care on Diabetes Quality of Care. J Gen Intern Med. 2021. https://doi.org/10.1007/s11606-020-06547-x

21. MedPAC. Telehealth in Medicare after the Coronavirus Public Health Emergency.; 2021. http://www.medpac.gov/docs/default-source/reports/mar21_medpac_report_ch14_sec.pdf?sfvrsn=0.

22. CY 2022 Medicare Physician Fee Schedule Proposed Rule. Centers for Medicare \& Medicaid Services2. https://www.cms.gov/Medicare/Medicare-Fee-for-Service-Payment/PhysicianFeeSched. Published 2021.

23. Centers for Medicare \& Medicaid Services. CMS Diabetes Quality Composite Measure. https://www.federalregister.gov/articles/2014/11/ 13/2014-26183/medicare- program-revisions-to-payment-policies-under-the-physician-fee-schedule- clinical-laboratory\#p-1915. Published 2021.

24. Health D of P. COUNTY OF LOS ANGELES DEPARTMENT OF PUBLIC HEALTH ORDER OF THE HEALTH OFFICER SAFER AT HOME ORDER FOR CONTROL OF COVID-19 Temporary Prohibition of All Events and Gatherings Closure of Non-Essential Businesses and Areas. 2020;(Cdc):1-11.

25. CMS Measures Inventory Tool - Diabetes Composite.

26. Daniel Croymans, Ian Hurst MH. Telehealth: The Right Care, at the Right Time, via the Right Medium. NEJM Catal. 2020;1(6):1-12. https://doi. org/10.1056/CAT.20.0564

27. Powers BW, Drzayich Antol D, Zhao Y, et al. Association Between Primary Care Payment Model and Telemedicine Use for Medicare Advantage Enrollees During the COVID-19 Pandemic. JAMA Heal Forum. 2021;2(7):e211597-e211597. https://doi.org/10.1001/ jamahealthforum.2021.1597

28. Patel SY, McCoy R, Barnett ML, Shah ND, Mehotra A. Diabetes Care and Glycemic Control During the COVID-19 Pandemic in the United States. JAMA Intern Med. 2021:2019-2021.

Publisher's Note: Springer Nature remains neutral with regard to jurisdictional claims in published maps and institutional affiliations. 